\title{
Can OPERA help in constraining neutrino non-standard interactions?
}

\author{
Andreu Esteban-Pretel ${ }^{* \dagger}$ \\ AHEP Group, Institut de Física Corpuscular - C.S.I.C./Universitat de València \\ Edificio Institutos de Paterna, Apt 22085, E-46071 Valencia, Spain \\ E-mail: andreu.esteban@ific.uv.es
}

\section{Patrick Huber}

Theory Division, Department of Physics, CERN

CH-1211 Geneva 23, Switzerland and

Institute for Particle, Nuclear and Astronomical Sciences,

Physics Department, Virgina Tech, Blacksburg, VA 24062, USA

E-mail: pahuber@vt.edu

\section{Jose W. F. Valle}

AHEP Group, Institut de Física Corpuscular - C.S.I.C./Universitat de València Edificio Institutos de Paterna, Apt 22085, E-46071 Valencia, Spain

E-mail: valledific.uv.es

\begin{abstract}
We study how much the unique ability of the OPERA experiment to directly detect $v_{\tau}$ can help in probing new, non-standard contact interactions of the third family of neutrinos. We perform a combined analysis of future, high-statistics MINOS and OPERA data. For the case of nonstandard interactions in $v_{\mu}$ to $v_{e}$ transitions we also include the impact of possible Double Chooz data. In all cases we find that the $v_{\tau}$ sample of OPERA is too small to be statistically significant. OPERA's real benefit for this measurement lies in its very high neutrino energy and hence very different $L / E$ compared to MINOS.
\end{abstract}

10th International Workshop on Neutrino Factories, Super beams and Beta beams June 30 - July 52008

Valencia, Spain

\footnotetext{
* Speaker.

${ }^{\dagger}$ Work supported by Spanish grants FPA2005-01269 (MEC) and ACOMP07/270 (Generalitat Valenciana) and by the European Commission RTN Contract MRTN-CT-2004-503369.
} 


\section{Introduction}

The confirmation of the neutrino oscillation interpretation of solar and atmospheric neutrino data by reactor and accelerator neutrino experiments brings a unique picture of neutrino physics in terms of three-neutrino oscillations [1], leaving little room for other non-standard neutrino properties. Nevertheless, it has long been recognized that any gauge theory of neutrino mass generation inevitably brings in dimension-6 non-standard neutrino interaction (NSI) terms. They can be of two types: flavor-changing (FC) and non-universal (NU) and their strength $\varepsilon G_{F}$ is highly modeldependent but may lie within the sensitivities of currently planned experiments.

The issue of NSI and oscillation in neutrino experiments with terrestrial sources has been studied in a large number of publications, for a recent list see references in [2]. In [3] it was shown that MINOS [4] on its own is not able to put new constraints on NSI parameters. On the other hand, in [5] the combination of atmospheric data with MINOS was proven to be effective in probing at least some of the NSI parameters.

The question we have addressed in [2] is whether the combination of MINOS and OPERA [6] can provide useful information on NSI. The idea is that OPERA will be able to detect $v_{\tau}$ and has a very different $L / E$ than MINOS. Both factors are known to help distinguishing NSI from oscillation effects. In [2] we focus on the simple case where NSI only affects neutrino propagation.

\section{Basic Setup}

Adding NSI into the propagation of neutrinos yields the following evolution Hamiltonian

$$
\mathscr{H}=\frac{1}{2 E} U\left(\begin{array}{ccc}
0 & 0 & 0 \\
0 & \Delta m_{21}^{2} & 0 \\
0 & 0 & \Delta m_{31}^{2}
\end{array}\right) U^{\dagger}+\frac{A}{2 E}\left(\begin{array}{lll}
1 & 0 & 0 \\
0 & 0 & 0 \\
0 & 0 & 0
\end{array}\right)+\frac{A}{2 E}\left(\begin{array}{ccc}
0 & 0 & \varepsilon_{e \tau} \\
0 & 0 & 0 \\
\varepsilon_{e \tau} & 0 & \varepsilon_{\tau \tau}
\end{array}\right),
$$

where $A \equiv \sqrt{2} G_{F} N_{e} 2 E$ and we have assumed the $\varepsilon$ 's to be real for simplicity ${ }^{1}$. We have also made use of the fact that all $\varepsilon_{x \mu}$ are fairly well constrained and hence are expected not to play a significant role at leading order. The effect of $\varepsilon_{e e}$ is a re-scaling of the matter density and all experiments considered here are not expected to be sensitive to matter effects. Hence we will set $\varepsilon_{e e}=0$. Note, that the $\varepsilon$ as defined here, are effective parameters. At the level of the underlying Lagrangian describing the NSI, the NSI coupling of the neutrino can be either to electrons, up or down quarks. From a phenomenological point of view, however, only the (incoherent) sum of all these contributions is relevant. For simplicity, we chose to normalize our NSI to the electron abundance. The NSI coupling to up or down quark would need to be 3 times as strong to produce the same effect in oscillations.

\subsection{Experiments}

All numerical simulations have been done using the GLoBES software $[9,10]$. In order to include the effects of the NSI we have customized the package by adding a new piece to the Hamiltonian as shown in equation 2.1. We have considered three different experiments: MINOS, OPERA and Double Chooz [11], the main characteristics of which are summarized in table 1.

\footnotetext{
${ }^{1}$ Inclusion of phases has been considered in the literature, see, e.g., Ref. [7, 8]
} 


\begin{tabular}{|c|c|c|c|c|c|c|c|c|c|}
\hline Label & & $L$ & & $\left\langle E_{v}\right\rangle$ & power & $\overline{t_{\text {run }}}$ & \multicolumn{3}{|c|}{ channel } \\
\hline $\mathrm{MINOS}_{2}(\mathrm{M} 2)$ & 735 & $\mathrm{~km}$ & & $3 \mathrm{GeV}$ & $\overline{5 \times 10^{20} \mathrm{pot} / \mathrm{yr}}$ & $5 \mathrm{yr}$ & $v_{\mu}$ & $\longrightarrow$ & $v_{e, \mu}$ \\
\hline OPERA $(\mathrm{O})$ & 732 & $\mathrm{~km}$ & & $17 \mathrm{GeV}$ & $4.5 \times 10^{19} \mathrm{pot} / \mathrm{yr}$ & $5 \mathrm{yr}$ & $v_{\mu}$ & $\rightarrow$ & $v_{e, \mu, \tau}$ \\
\hline Double Chooz (DC) & $\begin{array}{r}0.2 \\
1.05\end{array}$ & $\begin{array}{l}\mathrm{km} \\
\mathrm{km}\end{array}$ & $\begin{array}{l}\text { (near) } \\
\text { (far) }\end{array}$ & $4 \mathrm{MeV}$ & $8.4 \mathrm{GW}$ & $5 \mathrm{yr}$ & $\bar{v}_{e}$ & $\rightarrow$ & $\bar{v}_{e}$ \\
\hline
\end{tabular}

Table 1: Main parameters of the experiments under study. For more details see [2].

Concerning the neutrino oscillation parameters used to calculate the simulated event rates, we have taken the values given in Ref. [1], unless stated otherwise: $\sin ^{2} \theta_{12}^{\text {true }}=0.32, \sin ^{2} \theta_{23}^{\text {true }}=0.5$, $\sin ^{2} \theta_{13}^{\text {true }}=0,\left(\Delta m_{21}^{2}\right)^{\text {true }}=+7.6 \times 10^{-5} \mathrm{eV}^{2},\left(\Delta m_{31}^{2}\right)^{\text {true }}=+2.4 \times 10^{-3} \mathrm{eV}^{2}, \delta_{C P}^{\text {true }}=0$.

\section{Results}

\subsection{Disappearance - Probing NU NSI $\left(\varepsilon_{\tau \tau}\right)$}

As it has been previously shown in [5, 3] the presence of NSI, notably $\varepsilon_{\tau \tau}$, substantially degrades the goodness of the determination of the "atmospheric" neutrino oscillation parameters from experiment. Indeed as shown in the left panel of figure 1 our calculation confirms the same effect, showing how the allowed region in the $\sin ^{2} \theta_{23}-\Delta m_{31}^{2}$-plane increases in the presence of NSI.

This figure is the result of a combined fit to simulated OPERA and MINOS data in terms of the "atmospheric" neutrino oscillation parameters, leaving the mixing angle $\theta_{13}$ to vary freely. The inner black dot-dashed curve corresponds to the result obtained in the pure oscillation case (no NSI). The solid, red curve corresponds to a fit leaving $\varepsilon_{\tau \tau}$ and $\varepsilon_{e \tau}$ free. There, one sees that the NSI effect is dramatic for large NSI magnitudes. However, such large values are in conflict with atmospheric neutrino data $[12,5]$. In contrast, for lower NSI strengths allowed by the atmospheric + MINOS data combination [5], say $\left|\varepsilon_{\tau \tau}\right|=1.5$, the NSI effect becomes much smaller.

In summary, the inclusion of OPERA data helps only for very large values of $\varepsilon_{\tau \tau}$ as can be seen also from the first line of table 2. These large values, however are already excluded by the combination of MINOS and atmospheric results [5]. The slight improvement by OPERA comes from the $v_{\mu}$ sample and is due the very different value of $L / E$ compared to MINOS.

\begin{tabular}{|c||c|c|c||c|c|c|c|}
\hline \hline \multicolumn{1}{|c||}{} & \multicolumn{3}{c||}{$\sin ^{2} \theta_{13}^{\text {true }}=0$} & \multicolumn{4}{c|}{$\sin ^{2} \theta_{13}^{\text {true }}=0.1$} \\
\hline & $\mathrm{M} 2$ & $\mathrm{O}$ & $\mathrm{M} 2+\mathrm{O}$ & $\mathrm{M} 2$ & $\mathrm{O}$ & $\mathrm{M} 2+\mathrm{O}$ & $\mathrm{M} 2+\mathrm{O}+\mathrm{DC}$ \\
\hline \hline$\varepsilon_{\tau \tau}$ & {$[-11.8,11.8]$} & {$[-11.0,11.0]$} & {$[-9.2,9.2]$} & {$[-11.2,12.0]$} & {$[-10.8,11.0]$} & {$[-8.7,9.6]$} & {$[-5.6,5.8]$} \\
\hline$\varepsilon_{e \tau}$ & {$[-2.3,1.0]$} & {$[-2.5,1.6]$} & {$[-2.0,1.0]$} & {$[-4.5,1.5]$} & {$[-5.0,1.8]$} & {$[-4.1,1.4]$} & {$[-0.7,0.5]$} \\
\hline$\Delta m_{31}^{2}$ & {$[2.2,4.9]$} & {$[2.0,5.3]$} & {$[2.2,4.0]$} & {$[2.2,5.0]$} & {$[2.0,5.2]$} & {$[2.2,4.2]$} & {$[2.3,2.9]$} \\
\hline $\sin ^{2} \theta_{23}$ & {$[0.07,0.93]$} & {$[0.07,0.93]$} & {$[0.11,0.89]$} & {$[0.08,0.93]$} & {$[0.08,0.94]$} & {$[0.12,0.91]$} & {$[0.22,0.80]$} \\
\hline \hline
\end{tabular}

Table 2: $95 \%$ C.L. allowed regions for $\varepsilon_{\tau \tau}, \varepsilon_{e \tau}, \Delta m_{31}^{2}$ and $\sin ^{2} \theta_{23}$ for two different values of $\sin ^{2} \theta_{13}^{\text {true }}$ and different sets of experiments. Each row is obtained marginalizing over the remaining parameters, plus $\theta_{13}$. 

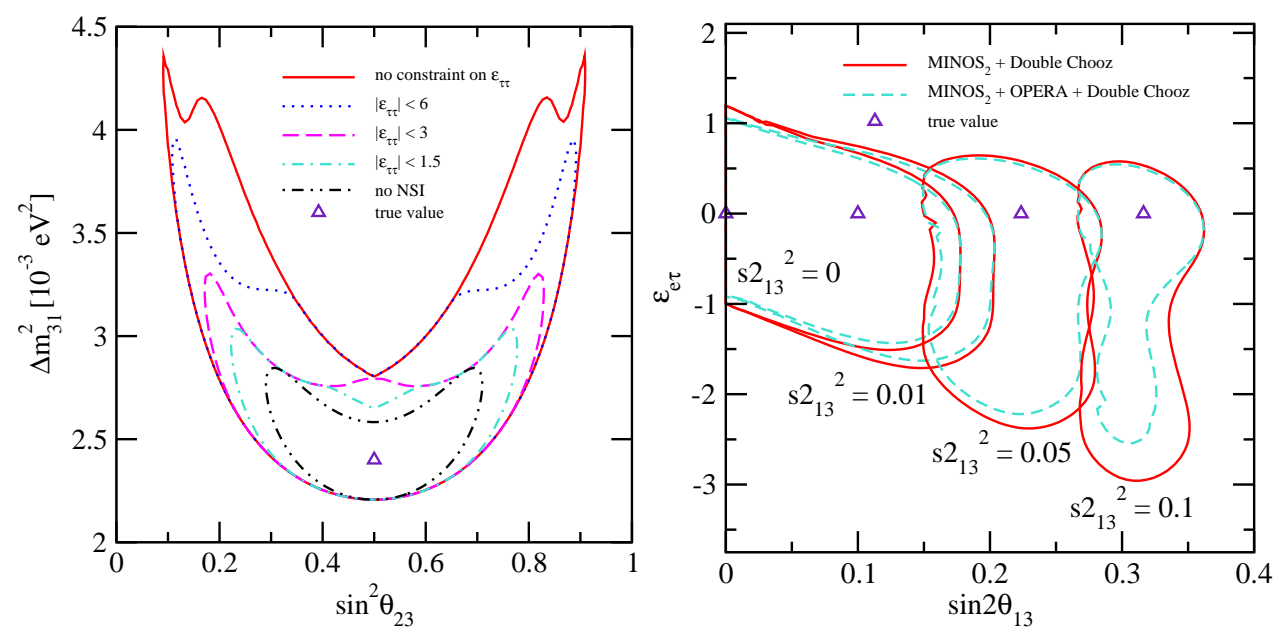

Figure 1: 95\% CL (2 dof) allowed regions. Left panel: $\theta_{13}, \varepsilon_{e}$ and $\varepsilon_{\tau \tau}$ left free. Right panel: fit for different combinations of the experiments and different true values of $\sin ^{2} 2 \theta_{13} . \Delta m_{31}^{2}, \theta_{23}$ and $\varepsilon_{\tau \tau}$ left free.

\subsection{Appearance - probing FC NSI $\left(\varepsilon_{\mathrm{e} \tau}\right)$}

It is well known that, in the presence of NSI, the determination of $\theta_{13}$ exhibits a continuous degeneracy [13] between $\theta_{13}$ and $\varepsilon_{e \tau}$ which leads to a drastic loss in sensitivity in $\theta_{13}$. In this context, it has been shown in [14], that even a very rudimentary ability to measure $P_{\mu \tau}$ may be sufficient to break this degeneracy. Therefore, it seems natural to ask whether OPERA can improve upon the sensitivity for $\varepsilon_{e \tau}$ that can be reached only with MINOS. The latter has been studied in [5] in combination with atmospheric neutrinos and on its own in Ref. [3]. The result, basically, was that MINOS will not be able to break the degeneracy between $\theta_{13}$ and $\varepsilon_{e \tau}$ and hence a possible $\theta_{13}$ bound from MINOS will, in reality, be a bound on a combination of $\varepsilon_{e \tau}$ and $\theta_{13}$.

In table 2 we display our results for a true value of $\sin ^{2} \theta_{13}=0$ and 0.1 and no NSI. The allowed range for $\varepsilon_{e \tau}$ shrinks only very little by the inclusion of OPERA data. Again, this result is not due to the $v_{\tau}$ sample but the different $L / E$ compared to MINOS.

In order to improve the sensitivity to NSI and to break the degeneracy between $\theta_{13}$ and $\varepsilon_{e \tau}$ it will be necessary to get independent information on either $\varepsilon_{e \tau}$ or $\theta_{13}$. We focus on $\theta_{13}$, because it is closer in time. Reactor experiments are very sensitive to $\theta_{13}$ but do not feel any influence from $\varepsilon_{e \tau}$ since the baseline is very short and the energy very low which leads to negligible matter effects. We consider here as new reactor experiment Double Chooz [11]. In the right panel of figure 1 we show the allowed regions in the $\sin 2 \theta_{13}-\varepsilon_{e \tau}$ plane for the combinations of MINOS and Double Chooz (red solid curves) and of MINOS, Double Chooz and OPERA (blue dashed curves) for four different input values of $\sin ^{2} 2 \theta_{13}$ as indicated in the plot. As expected, the effect of Double Chooz in all four cases is to constrain the allowed $\sin 2 \theta_{13}$ range. The impact of OPERA, given by the difference between the solid and dashed lines, is absent for very small true values of $\sin 2 \theta_{13}$ and increases with increasing true values. For the largest currently permissible values of $\theta_{13} \simeq 0.16$, OPERA can considerably reduce the size of the allowed region and help to resolve the degeneracy. In that parameter region a moderate increase in the OPERA exposure would make it possible to constrain large negative values of $\varepsilon_{e}$. Again, this effect has nothing to do with $v_{\tau}$ detection and, in this case, is based on the different $L / E$ in $v_{e}$-appearance channel. 


\section{Conclusion}

In [2] we have studied how OPERA can help in improving the sensitivities on neutrino nonstandard contact interactions of the third family of neutrinos. In our analysis we considered a combined OPERA fit together with high statistics MINOS data, in order to obtain restrictions on neutrino oscillation parameters in the presence of NSI. Due to its unique ability of detecting $v_{\tau}$ one would expect that the inclusion of OPERA data would provide new improved limits on the universality violating NSI parameter $\varepsilon_{\tau \tau}$. We found, however, that the $v_{\tau}$ data sample is too small to be of statistical significance. OPERA also has a $v_{\mu}$ sample, which can help constraining NSI. Here the effect is due to the very different $L / E$ of OPERA compared to MINOS. This makes the OPERA $v_{\mu}$ sample more sensitive to NSI. However, the improvement is small and happens in a part of the NSI parameter space which is essentially excluded by atmospheric neutrino data.

We have also studied the possibility of constraining the FC NSI parameter $\varepsilon_{e} \tau$. For this purpose it is crucial to have a good knowledge of $\theta_{13}$. Since reactor neutrino experiments are insensitive to the presence of NSI of the type considered here, they can provide a clean measurement of $\theta_{13}$. Therefore, we included future Double Chooz data. The conclusion for $\varepsilon_{e \tau}$ with respect to the $v_{\tau}$ sample is the same as before: the sample is very much too small to be of any statistical significance. OPERA's different $L / E$ again proves to be its most important feature and allows to shrink the allowed region on the $\sin ^{2} \theta_{13}-\varepsilon_{e \tau}$ plane for large $\theta_{13}$ values. Here a modest increase in OPERA exposure would allow to completely lift the $\theta_{13}-\varepsilon_{e \tau}$ degeneracy and thus to obtain a unique solution.

\section{References}

[1] T. Schwetz, M. Tortola and J. W. F. Valle, arXiv:0808.2016 [hep-ph].

[2] A. Esteban-Pretel, J. W. F. Valle and P. Huber, arXiv:0803.1790 [hep-ph].

[3] M. Blennow, T. Ohlsson and J. Skrotzki, hep-ph/0702059.

[4] MINOS collaboration, D. G. Michael et al., Phys. Rev. Lett. 97, 191801 (2006), [hep-ex/0607088].

[5] A. Friedland and C. Lunardini, Phys. Rev. D74, 033012 (2006), [hep-ph/0606101].

[6] OPERA collaboration, M. Guler et al., (2000), CERN-SPSC-2000-028.

[7] T. Ota, J. Sato and N.-a. Yamashita, Phys. Rev. D65, 093015 (2002), [hep-ph/0112329].

[8] M. Blennow, D. Meloni, T. Ohlsson, F. Terranova and M. Westerberg, arXiv:0804.2744 [hep-ph].

[9] P. Huber, M. Lindner and W. Winter, Comput. Phys. Commun. 167, 195 (2005), [hep-ph/0407333].

[10] P. Huber, J. Kopp, M. Lindner, M. Rolinec and W. Winter, Comput. Phys. Commun. 177, 432 (2007), [hep-ph/0701187].

[11] Double Chooz collaboration, F. Ardellier et al., hep-ex/0606025.

[12] N. Fornengo et al., Phys. Rev. D65, 013010 (2002), [hep-ph/0108043].

[13] P. Huber, T. Schwetz and J. W. F. Valle, Phys. Rev. Lett. 88, 101804 (2002), [hep-ph/0111224].

[14] M. Campanelli and A. Romanino, Phys. Rev. D66, 113001 (2002), [hep-ph/0207350]. 